\title{
Trace Determination of Palladium in Environmental Samples by Adsorptive Voltammetry
}

\author{
C. L. Rojas* \\ Centro de Electroquímica y Energía Química, Escuela de Química, \\ Universidad de Costa Rica, 2060 San Pedro, Montes de Oca, Costa Rica
}

Received May 21, 2004; accepted in revised form March 3, 2005

\begin{abstract}
The objective of the present work is the study and optimization of a voltammetric methodology to determine palladium traces and ultratraces, with the purpose of being able to establish the environmental levels of this element and to verify if a contamination takes place starting from the gases of escape of the vehicles equipped with catalytic converters. The voltammetric determination of palladium is based in the formation of a complex of palladium with dimethylglyoxime that adsorbes actively on the surface of a hanging mercury drop electrode, for then to be reduced when making the square wave potential sweep. The quantification is made by the method of standard additions. The study of interferences demonstrated that $\mathrm{Na}^{+}, \mathrm{Mg}^{2+}, \mathrm{K}^{+}, \mathrm{Ca}^{2+}, \mathrm{Zn}^{2+}, \mathrm{Cu}^{2+}$ and $\mathrm{Al}^{3+}$ are not even interferences at the level of $10 \mathrm{mg} / \mathrm{L}$. The detection and quantification limits of the methodology are respectively $10 \mathrm{ng} / \mathrm{L}$ and $33 \mathrm{ng} / \mathrm{L}$, with a sensibility of $(4.3 \pm 0.8) * 10^{2} \mathrm{nA} \mathrm{L} / \mathrm{ng}$.
\end{abstract}

Keywords: palladium, ultratraces, voltammetry, determination, environmental.

\section{Introducción}

Los metales del grupo del platino han ganado mucha importancia en los últimos años como resultado del desarrollo tecnológico, principalmente en el área de la catálisis. Se encuentran en muy pequeñas cantidades en el medio ambiente, en los alimentos y en los seres vivos, así como en comparación con los demás metales pesados. $\mathrm{Su}$ toxicidad es significativa sólo en circunstancias muy

\footnotetext{
* Corresponding author. E-mail address: cleon@equi.ucr.ac.cr
} 
particulares. Sin embargo, su inhalación o el contacto con aerosoles de algunos de sus compuestos pueden provocar reacciones alérgicas, dermatitis, dificultades respiratorias y otros síntomas. Aun así, se conoce poco sobre los efectos biológicos de los elementos del grupo del platino y sus compuestos $[1,2]$.

El establecimiento de los niveles naturales de los elementos del grupo del platino, es el primer paso antes de poder establecer cualquier relación epidemiológica entre estos elementos y cualquier enfermedad. De esta forma, será posible determinar después cualquier incremento de concentración producida por fenómenos antropogénicos [3].

La introducción en los años 80 de los convertidores catalíticos en los coches, ha provocado un aumento constante de las concentraciones ambientales de platino, rodio y paladio. Aparte de los beneficios indiscutibles que los convertidores catalíticos tienen para el medio ambiente, ya que reducen los niveles de gases emitidos por los coches, el aumento de los EGP en el ambiente ha provocado una seria discusión sobre los posibles efectos adversos de estos elementos sobre la salud [3-10]. Por ello, en los últimos años se han efectuado un gran número de estudios encaminados a determinar los niveles ambientales de los elementos del grupo del platino y establecer de esta forma el grado de contaminación existente.

Raber y colaboradores [10] investigaron una metodología voltamperométrica utilizando un electrodo de pasta de carbono modificado con tioridazina. Obtuvieron un límite de detección de $500 \mathrm{ng} / \mathrm{L}$ al utilizar un tiempo de preconcentración de 120 segundos. Además, el método presenta pocas interferencias.

La determinación voltamperométrica de paladio se fundamenta en la formación de un complejo del paladio con la dimetilglioxima, que se adsorbe activamente sobre la superficie de un electrodo de gota suspendida de mercurio. La dimetilglioxima reacciona con el paladio de acuerdo con las siguientes ecuaciones:

$$
\begin{gathered}
\operatorname{Pd}\left(\mathrm{H}_{2} \mathrm{O}\right)^{2+}{ }_{\mathrm{n}} \leftrightarrow \mathrm{Pd}(\mathrm{OH})^{+} \leftrightarrow \mathrm{Pd}(\mathrm{OH})_{2} \text { (dis.) } \\
\mathrm{Pd}(\mathrm{OH})_{2}(\text { dis. })+2 \mathrm{H}_{2} \mathrm{D} \leftrightarrow \mathrm{Pd}(\mathrm{HD})_{2}+2 \mathrm{H}_{2} \mathrm{O}
\end{gathered}
$$


y es este complejo el que se adsorbe sobre el electrodo y luego es reducido al efectuar el barrido de potencial.

\section{Parte Experimental}

Para la realización de este estudio se utilizó un equipo EG\&G modelo 384-B acoplado a un sistema de electrodos también EG\&G, modelo 303-A, que consiste de un electrodo de gota suspendida de mercurio como electrodo de trabajo, un electrodo de referencia de plata/cloruro de plata y un electrodo auxiliar de carbono vitrificado. Todos los reactivos químicos así como el agua utilizados fueron calidad ultrapura.

Para la optimización de las condiciones experimentales, se consideraron inicialmente las condiciones utilizadas por Georgieva y Pihlar [11] (Tabla 1), así como las condiciones básicas con que cuenta el polarógrafo (Tabla 2) y a partir de ellas, se hizo la optimización.

Tabla 1. Condiciones voltamperométricas utilizadas por Georgieva y Pihlar para la determinación de paladio.

\begin{tabular}{|c|c|}
\hline Condición & Valor \\
\hline Técnica & Pulso diferencial \\
\hline Electrolito soporte & Tampón acetato $0.1 \mathrm{~mol} / \mathrm{L} \mathrm{pH} \mathrm{3.6}$ \\
\hline Dimetilglioxima & $1 \times 10^{-4} \mathrm{~mol} / \mathrm{L}$ \\
\hline Potencial inicial & $-0.3 \mathrm{~V}(\mathrm{vs} . \mathrm{Ag} / \mathrm{AgCl})$ \\
\hline Tiempo de preadsorción & $5 \mathrm{minutos}$ \\
\hline Velocidad & $8 \mathrm{mV} / \mathrm{s}$ \\
\hline Tamaño de la gota & Mediana $\left(0.025 \mathrm{~cm}^{2}\right)$ \\
\hline
\end{tabular}

Tabla 2. Otras condiciones voltamperométricas iniciales utilizadas.

\begin{tabular}{|c|c|}
\hline Condición & Valor \\
\hline Altura del pulso & $-50 \mathrm{mV}$ \\
\hline Incremento de barrido & $5 \mathrm{mV}$ \\
\hline Tiempo entre pulsos & $0.25 \mathrm{~s}$ \\
\hline
\end{tabular}

La optimización de un parámetro se realizó fijando todos los parámetros menos el que se optimiza a valores determinados y variando el parámetro en estudio. 
Para cada modificación, se obtuvieron voltamperogramas y se consideró como valor óptimo aquel en el que se obtenía la mayor señal para paladio.

\section{Resultados y discusión}

\section{Selección de la técnica voltamperométrica}

La correcta selección de la técnica voltamperométrica es importante, pues de ello dependen tanto la sensibilidad como los parámetros instrumentales que después será necesario optimizar. Debido a las bajas concentraciones de paladio que se pretenden medir, se hace necesario utilizar una técnica voltamperométrica que permita realizar una preconcentración del analito o de un complejo del mismo, sobre una gota de mercurio de un electrodo de gota suspendida. De las ocho técnicas voltamperométricas con que cuenta el polarógrafo utilizado, sólo dos permiten obtener voltamperogramas con preconcentración previa: diferencial de pulso y onda cuadrada. Los resultados obtenidos se muestran en la Fig. 1.

Tabla 3. Condiciones voltamperométricas utilizadas para la determinación de paladio.

\begin{tabular}{|c|c|}
\hline Condición & Valor \\
\hline Técnica & Onda cuadrada \\
\hline Electrolito soporte & Tampón acetato $0.1 \mathrm{~mol} / \mathrm{L} \mathrm{pH} \mathrm{3.6}$ \\
\hline Dimetilglioxima & $1 \times 10^{-4} \mathrm{~mol} / \mathrm{L}$ \\
\hline Potencial inicial & $-0.3 \mathrm{~V}(\mathrm{vs} . \mathrm{Ag} / \mathrm{AgCl})$ \\
\hline Tiempo de preadsorción & $5 \mathrm{minutos}$ \\
\hline Frecuencia & $50 \mathrm{~Hz}$ \\
\hline Altura del pulso & $-50 \mathrm{mV}$ \\
\hline Incremento de barrido & $5 \mathrm{mV}$ \\
\hline Tamaño de la gota & Mediana $\left(0.025 \mathrm{~cm}^{2}\right)$ \\
\hline
\end{tabular}



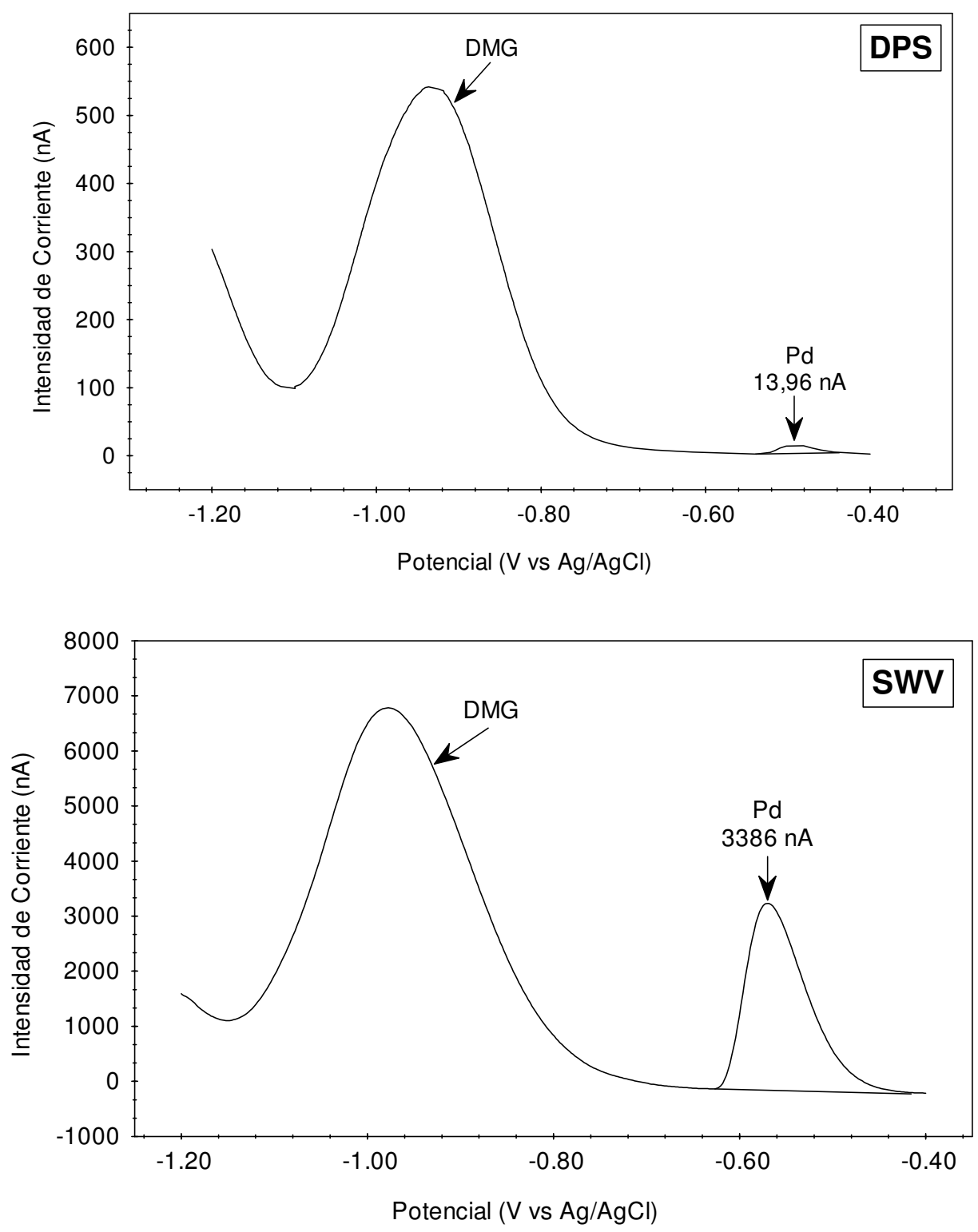

Figura 1. Voltamperogramas de $10 \mu \mathrm{g} / \mathrm{L}$ de paladio utilizando dos técnicas diferentes: voltamperometría de redisolución con diferencial de pulso (DPS) y voltamperometría de redisolución con onda cuadrada (SWV).

En la Fig. 1 se observa claramente el aumento de sensibilidad que se obtiene al cambiar, para la misma disolución, la técnica voltamperométrica de diferencial de pulso a onda cuadrada (13.96 nA vs. 3386 nA). Por lo tanto, se seleccionó como técnica a emplear en el resto de este estudio la voltamperometría de onda cuadrada con redisolución, con las condiciones instrumentales que se muestran en la Tabla 3, a menos que se indique lo contrario. 


\section{Optimización del pH del electrolito soporte}

Para la optimización de este parámetro, se prepararon tampones acetato de diferente $\mathrm{pH}$, manteniendo la concentración de ion acetato constante en 0.1 $\mathrm{mol} / \mathrm{L}$. Para ello se preparó una disolución de ácido acético $0.1 \mathrm{~mol} / \mathrm{L}$ y otra de acetato de sodio también $0.1 \mathrm{~mol} / \mathrm{L}$. Los tampones se prepararon mezclando diferentes cantidades de cada una de estas dos disoluciones. Los resultados se muestran en la Fig. 2.

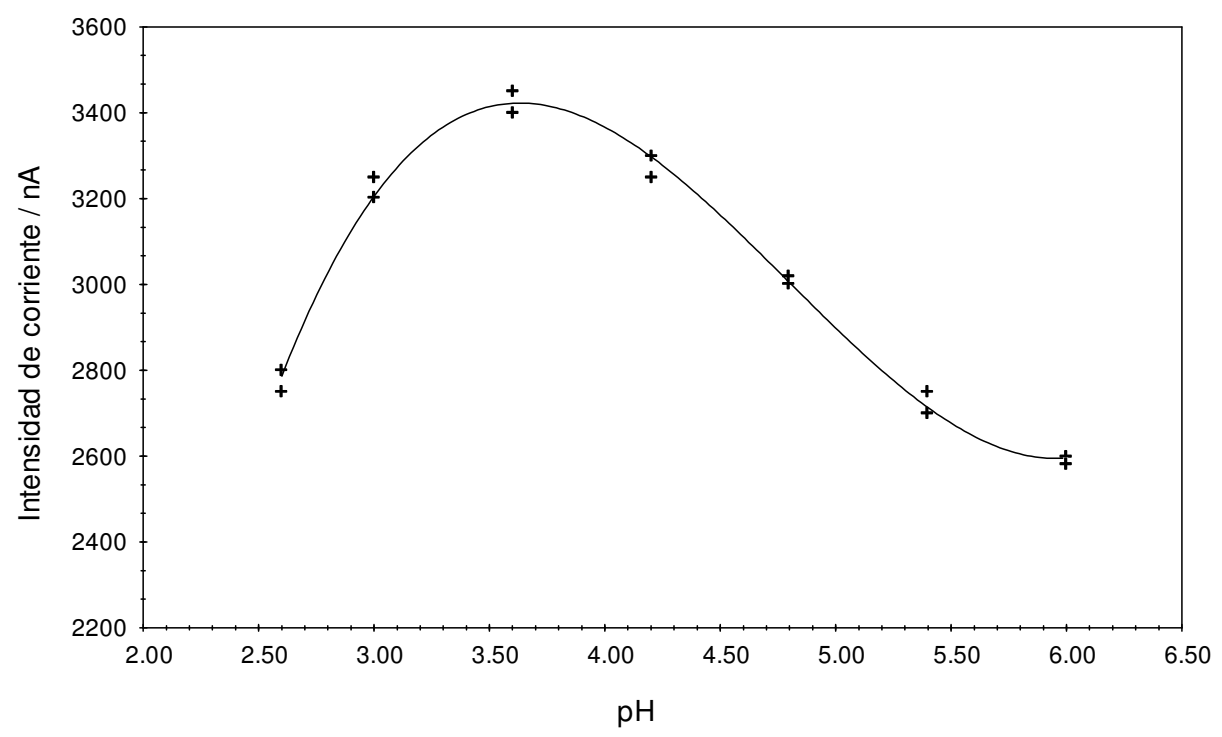

Figura 2. Dependencia con el $\mathrm{pH}$ de la sensibilidad de la determinación voltamperométrica de $10.0 \mu \mathrm{g} / \mathrm{L}$ de paladio.

Se puede observar como el valor máximo de corriente se alcanza para un $\mathrm{pH}$ de 3.6. Esto concuerda con la estequiometría planteada, en donde es la dimetilglioxima protonada la que forma el complejo con el paladio. Para los estudios posteriores se usará como óptimo un tampón acetato $(0.1 \mathrm{~mol} / \mathrm{L})$ con un $\mathrm{pH}$ de 3.6.

\section{Optimización de la concentración de dimetilglioxima}

En voltamperometría adsortiva con un complejante que reacciona con el analito, es importante optimizar la concentración del ligando, de tal forma que la misma 
sea lo suficientemente alta como para permitir el análisis, pero además, que no sea tan elevada como para que el mismo complejante se comporte como una interferencia. Hay que recordar, que la gran mayoría de los complejantes utilizados en química analítica son capaces de adsorberse por si mismos sobre la superficie de un electrodo y por ello es que se puede comportar también como una interferencia, al bloquear los sitios activos sobre el electrodo. Los resultados obtenidos se muestran en la Fig. 3.

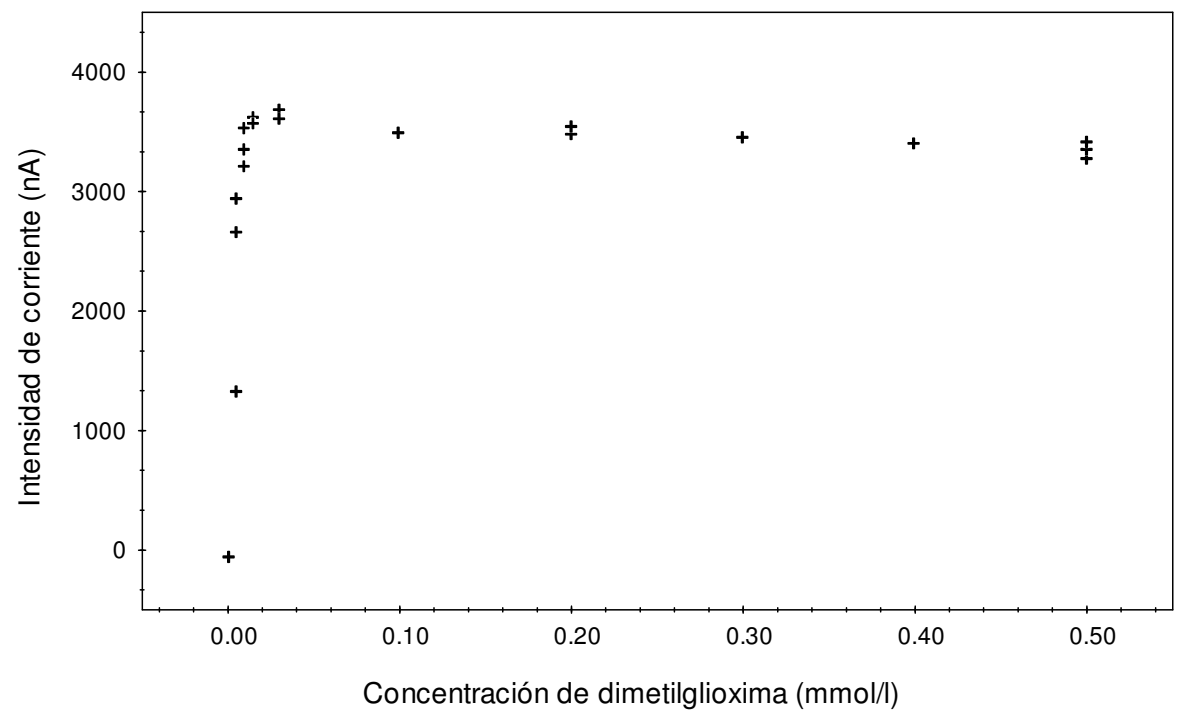

Figura 3. Optimización de la concentración de dimetilglioxima en el electrolito soporte, sobre el análisis de $10.0 \mu \mathrm{g} / \mathrm{L}$ de paladio.

Los resultados muestran como la señal aumenta con la concentración de la dimetilglioxima, hasta una concentración aproximada de $0.01 \mathrm{mmol} / \mathrm{L}$, después de la cual la señal se estabiliza en un valor casi constante, aunque se aprecia una pequeña disminución de la corriente. Por lo tanto, se decidió seguir utilizando como concentración óptima de dimetilglioxima $0.10 \mathrm{mmol} / \mathrm{L}$, ya que ésta se encuentra en la meseta observada en la Fig. 3 y garantizándose con ello que una pequeña variación de la concentración no producirá un efecto negativo sobre el análisis. 


\section{Influencia del tamaño de la gota}

El electrodo utilizado tiene predefinidos tres tamaños de gota que pueden ser utilizados cuando se trabaja con el electrodo de gota suspendida de mercurio: pequeña (S), mediana (M) y grande (L) $\left(0.0024 \mathrm{~cm}^{2}, 0.0039 \mathrm{~cm}^{2}\right.$ y $0.0060 \mathrm{~cm}^{2}$, respectivamente). Dependiendo del tamaño de la gota, así es el tamaño de la superficie del electrodo expuesta a la disolución y por lo tanto, de ella depende el número de sitios activos que pueden ser utilizados para preconcentrar un analito. A cada uno de los tres tamaños de gota, se obtuvieron los voltamperogramas correspondientes. Los resultados obtenidos se muestran en la Fig. 4.

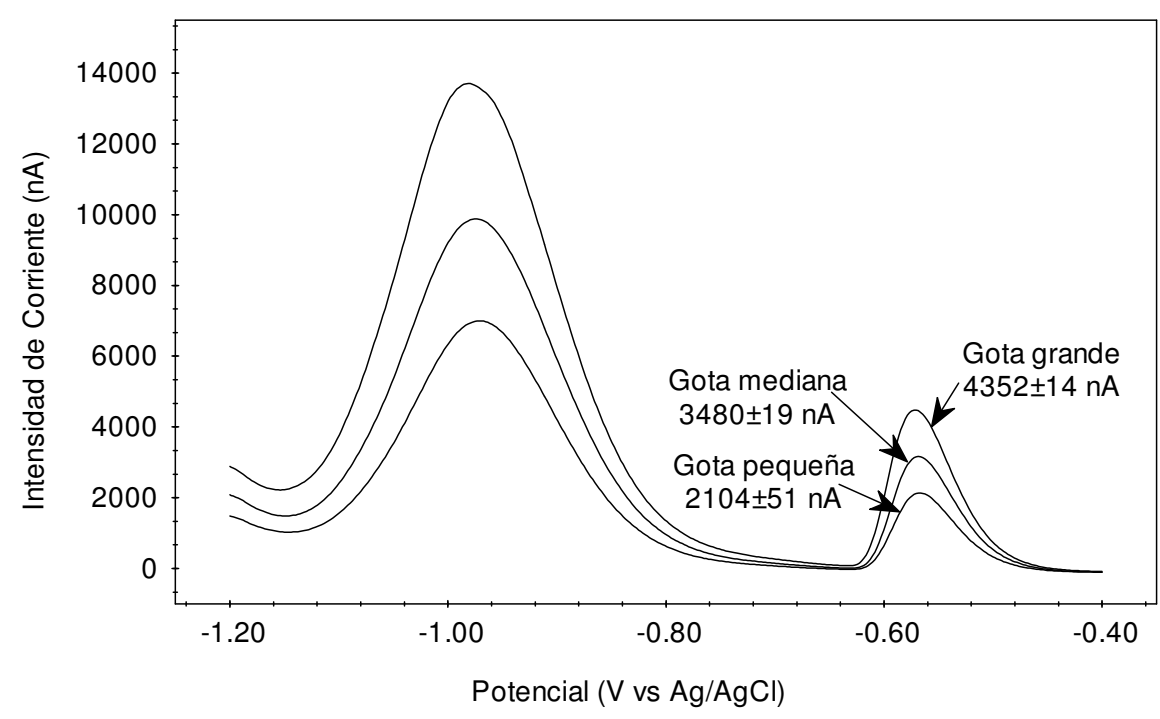

Figura 4. Variación de la señal voltamperométrica de $10.0 \mu \mathrm{g} / \mathrm{L}$ de paladio con el tamaño de la gota del electrodo de gota suspendida de mercurio.

Es evidente en los resultados obtenidos, que cuanto mayor es el tamaño de la gota, mayor es la corriente obtenida para la señal del paladio, obteniéndose un aumento de sensibilidad del $52 \%$ al pasar de la gota pequeña a la mediana, del $38 \%$ al pasar de la mediana a la grande y del $110 \%$ al pasar de la pequeña a la grande directamente. Para los estudios posteriores se seguirá usando el tamaño de gota grande. 


\section{Influencia de la frecuencia de la onda cuadrada}

En voltamperometría de onda cuadrada, la frecuencia de la onda define dos parámetros importantes, la velocidad de barrido de potencial y el tiempo entre pulsos. Los resultados se muestran en la Fig. 5.

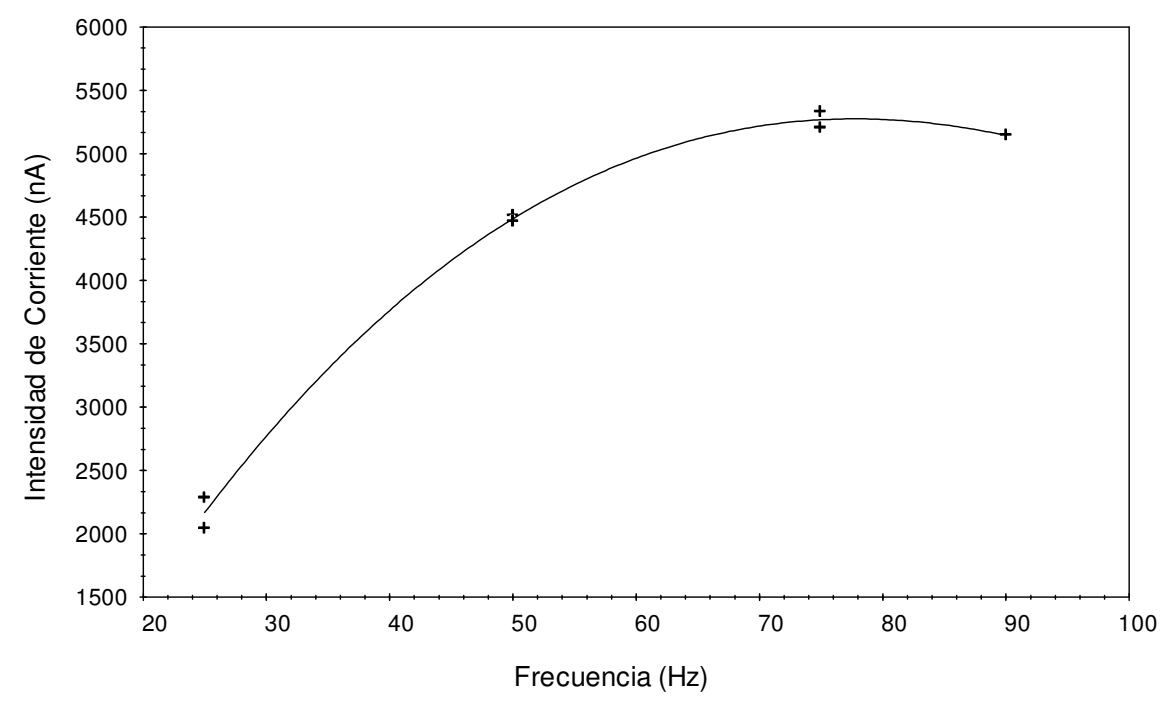

Figura 5. Optimización de la frecuencia en onda cuadrada para el análisis de $10.0 \mu \mathrm{g} / \mathrm{L}$ de paladio.

Como se puede observar, la señal aumenta con la frecuencia de la onda cuadrada, alcanzando el máximo en $75 \mathrm{~Hz}$. El máximo valor que puede tomar este parámetro en el polarógrafo utilizado, $100 \mathrm{~Hz}$, no se puede utilizar debido a que los voltamperogramas presentan un ruido de fondo considerable, en forma de pequeñas oscilaciones de la corriente. Lo mismo ocurre a $90 \mathrm{~Hz}$, que es el último punto de la gráfica y que también presenta un ruido considerable. A $75 \mathrm{~Hz}$, si bien las corrientes son mayores, se tiene una baja reproducibilidad de las señales, por lo que no se recomienda su uso. Se selecciona por lo tanto una frecuencia de $50 \mathrm{~Hz}$ como la óptima, ya que que se obtienen buenas señales y buena sensibilidad.

\section{Influencia del incremento de potencial}

Junto con la frecuencia, este parámetro define la velocidad de barrido de potencial en la voltamperometría de onda cuadrada e influye también de forma 
significativa sobre la resolución que tienen los voltamperogramas. Los resultados se muestran en la Fig. 6.

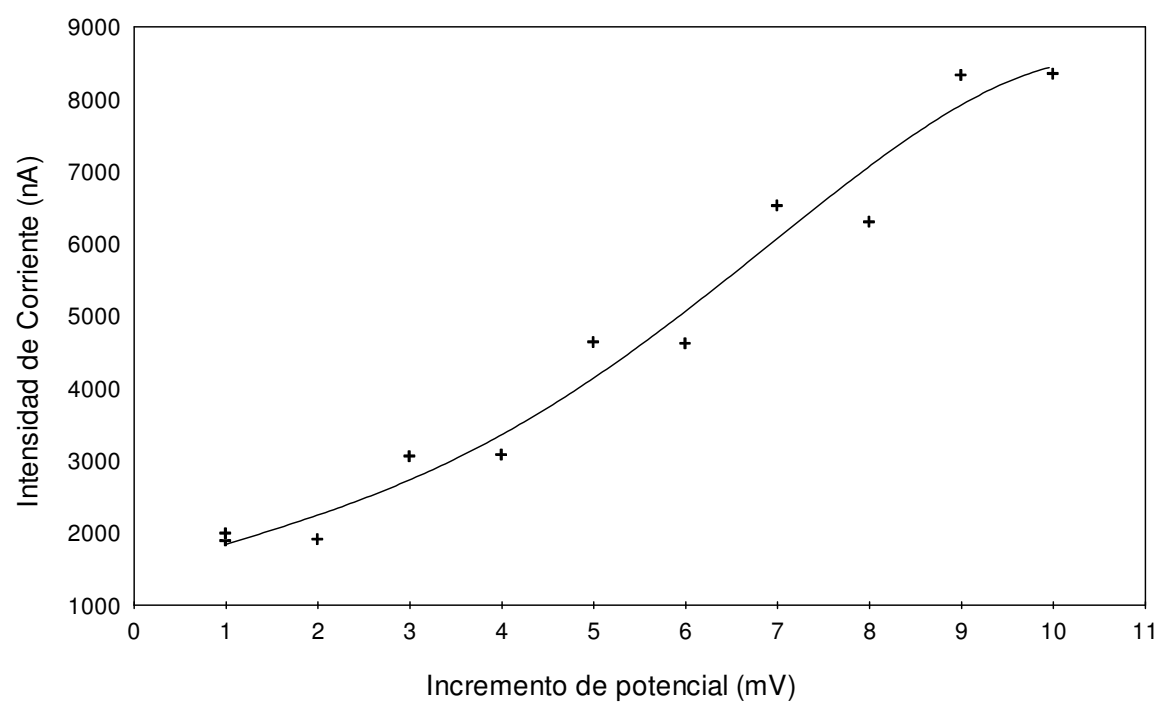

Figura 6. Optimización del incremento de potencial para el análisis de $10.0 \mu \mathrm{g} / \mathrm{L}$ de paladio.

La intensidad de corriente de la señal de paladio aumenta progresivamente con el aumento de este parámetro, hasta llegar a un máximo en $10 \mathrm{mV}$. En los resultados mostrados en la Fig. 6 se observa como la intensidad de corriente aparenta crecer en "escalones", repitiéndose casi el mismo valor de corriente al pasar de un incremento de potencial con un valor impar a otro con un valor par. No se ha encontrado ni en la literatura ni en los manuales de operación del polarógrafo ninguna referencia que pueda explicar este comportamiento. En todo caso, se decidió utilizar $10 \mathrm{mV}$ como valor óptimo para este parámetro.

\section{Influencia del potencial de preadsorción}

En la Fig. 7 se muestran los resultados correspondientes a la optimización del potencial de preadsorción. El potencial de preadsorción se varió entre 0 y $-0.45 \mathrm{~V}$ y para cada potencial se obtuvieron los voltamperogramas correspondientes. 


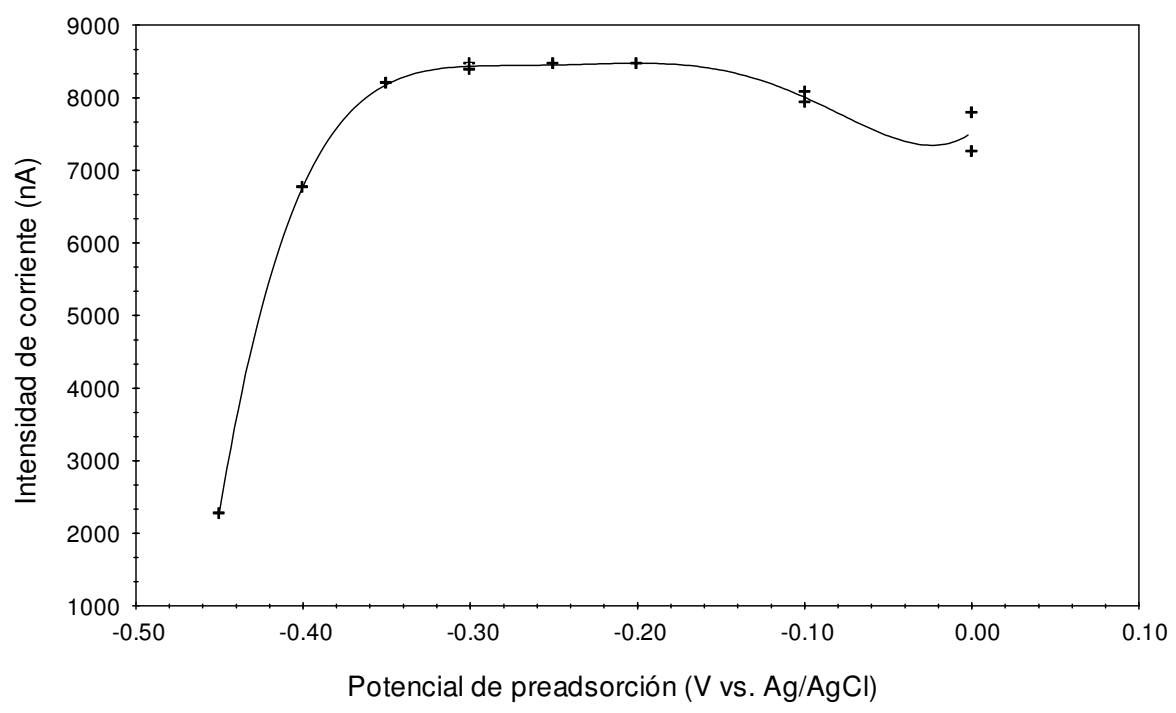

Figura 7. Optimización del potencial de preadsorción para el análisis de $10.0 \mu \mathrm{g} / \mathrm{L}$ de paladio.

Como se observa en la Fig. 7, la altura de la señal del paladio no varía mucho cuando se cambia el potencial de preadsorción desde cero hasta $-0.30 \mathrm{~V}$, después del cual se observa una fuerte disminución de la señal, posiblemente por la cercanía al potencial al cual aparece la propia señal del dimetilglioximato de paladio. Se escogió como potencial óptimo $-0.30 \mathrm{~V}$, debido a que se encuentra en la meseta de la representación gráfica, lo que asegura que una pequeña fluctuación en el potencial no afectará significativamente la determinación.

\section{Influencia del tamaño del pulso}

Una gran parte de la ganancia en sensibilidad que se obtiene al usar diferencial de pulso u onda cuadrada, se debe a la aplicación de pulsos de potencial, y la magnitud de la señal obtenida depende del tamaño del pulso utilizado, por lo que es necesaria la optimización de este parámetro. Los resultados se muestran en la Fig. 8. 


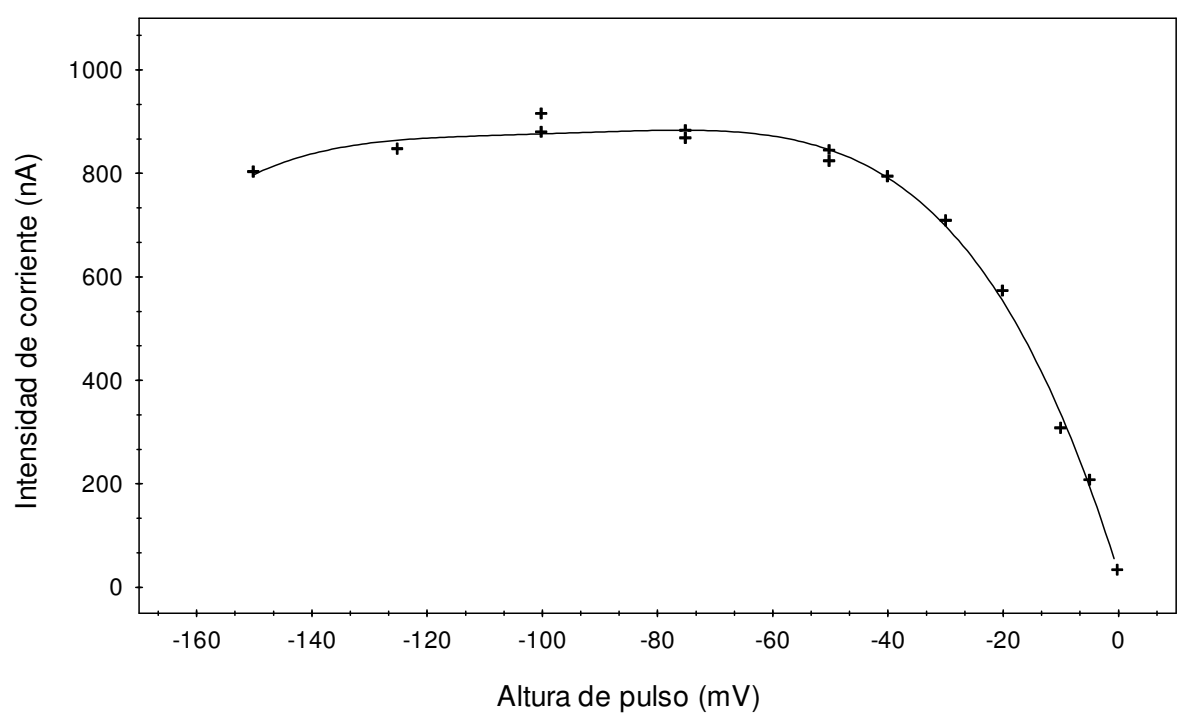

Figura 8. Optimización de la altura del pulso de potencial de la onda cuadrada sobre el análisis de $1.00 \mu \mathrm{g} / \mathrm{L}$ de paladio.

Se observa como la señal aumenta conforme aumenta la magnitud absoluta del pulso, llegando a estabilizarse a pulsos más negativos que $-50 \mathrm{mV}$. Por eso se decidió utilizar como óptimo una amplitud de pulso de $-75 \mathrm{mV}$, lo que garantiza una mayor estabilidad de la señal frente a pequeñas fluctuaciones del potencial.

\section{Influencia del tiempo de preadsorción}

El paso previo de preconcentración activa, es el que permite en última instancia la determinación de trazas de sustancias, pues permite un enriquecimiento in-situ de los analitos sobre el propio electrodo, donde son determinados. Debido a que la preconcentración no es exhaustiva, es requisito indispensable controlar estrictamente el tiempo de la preconcentración, para así poder obtener resultados reproducibles. Además, dependiendo del tiempo de preadsorción, así será la cantidad de analito preconcentrado sobre el electrodo y por ello es necesaria la optimización de este factor. Los resultados se muestran en la Fig. 9. 


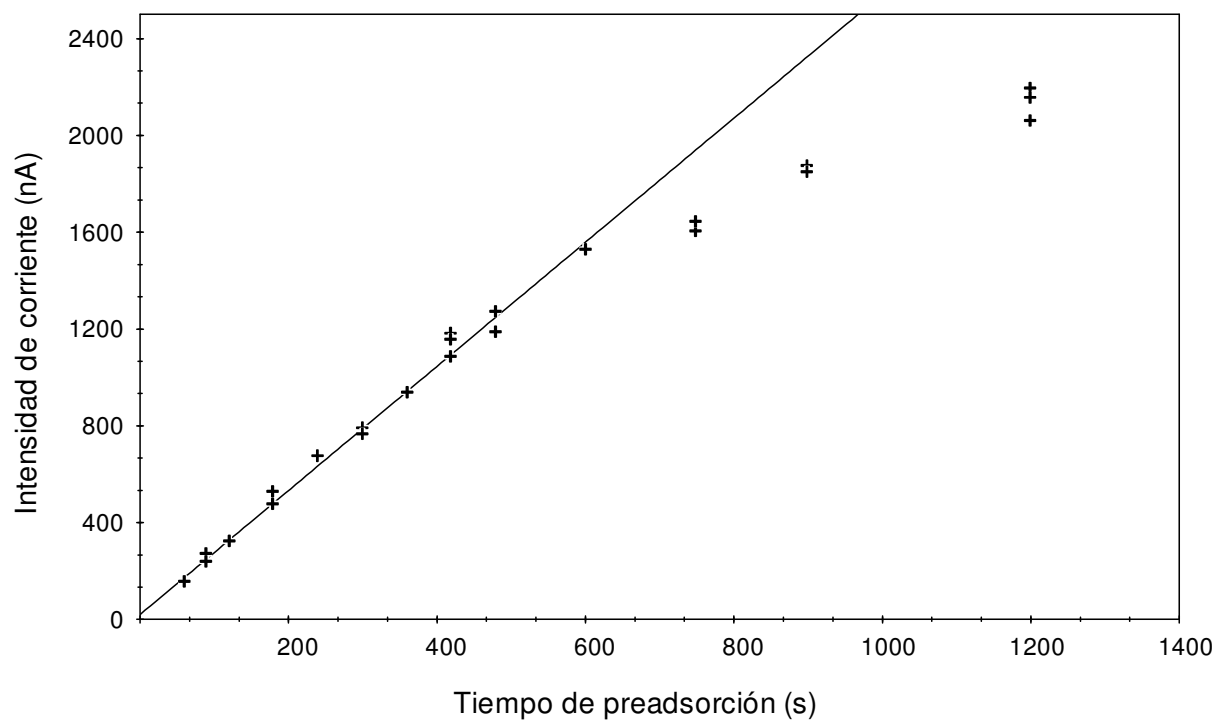

Figura 9. Optimización del tiempo de preadsorción sobre el análisis de $10.0 \mu \mathrm{g} / \mathrm{L}$ de paladio.

Como se puede observar, la señal crece linealmente con el tiempo de preadsorción hasta los 600 segundos y después, si bien sigue creciendo, lo hace con una sensibilidad mucho menor. Por lo tanto, se recomienda utilizar como tiempo de preadsorción uno igual o inferior a 600 segundos, dependiendo de la concentración de paladio que se mida. Para el resto de este estudio se decidió seguir utilizando $300 \mathrm{~s}$, con el fin de no prolongar excesivamente los análisis.

\section{Influencia del tiempo de reposo}

El tiempo de reposo después de la preadsorción se utiliza para asegurar que en la disolución no se presenten fenómenos de transporte de masas por convección, lo cual interfiere en las medidas voltamperométricas, pues estas se basan en la medida de corrientes debidas únicamente a procesos de difusión. Los resultados se muestran en la Fig. 10.

Como se puede observar, la señal del paladio cambia poco con el aumento del tiempo de reposo, aunque se observa una pequeña disminución de la intensidad de corriente. Si bien el máximo se obtiene para un tiempo de cero segundos, se prefirió seleccionar como óptimo 5 segundos. Dos razones existen para ello: 
- la disminución de la intensidad de corriente no es significativa al usar 5 y no cero segundos;

- 5 segundos aseguran el reposo de la disolución.

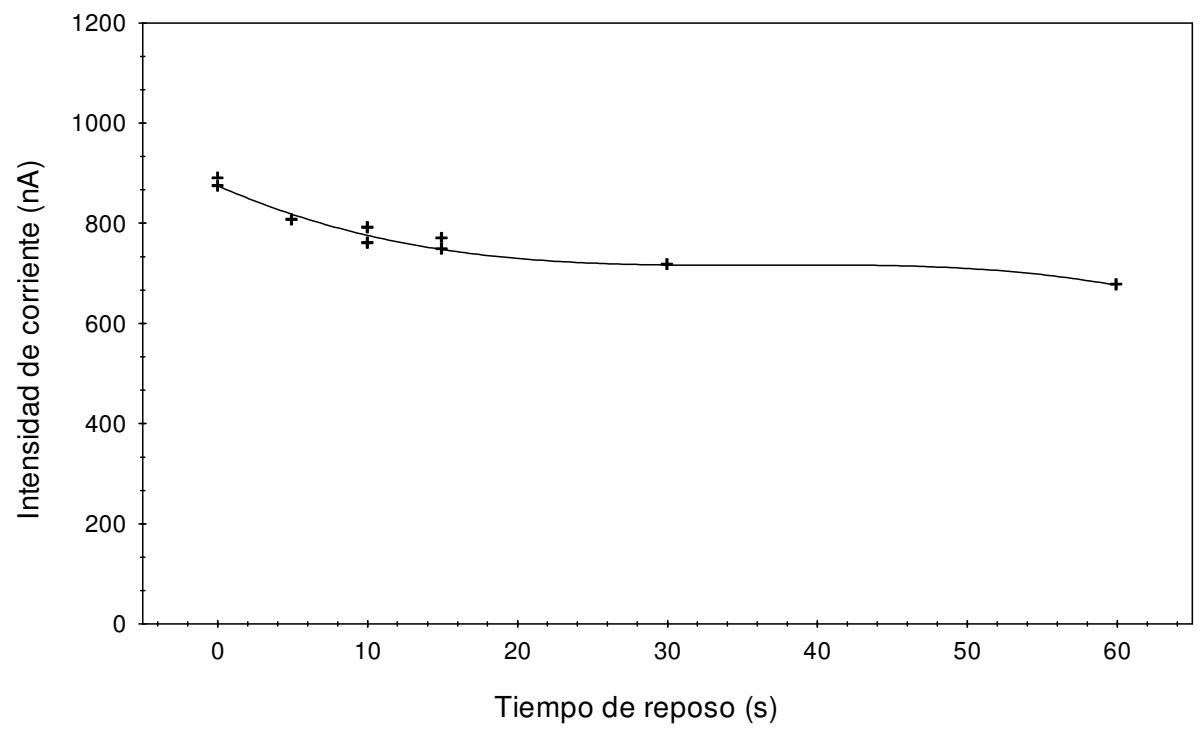

Figura 10. Optimización del tiempo de reposo para el análisis de $1.00 \mu \mathrm{g} / \mathrm{L}$ de paladio.

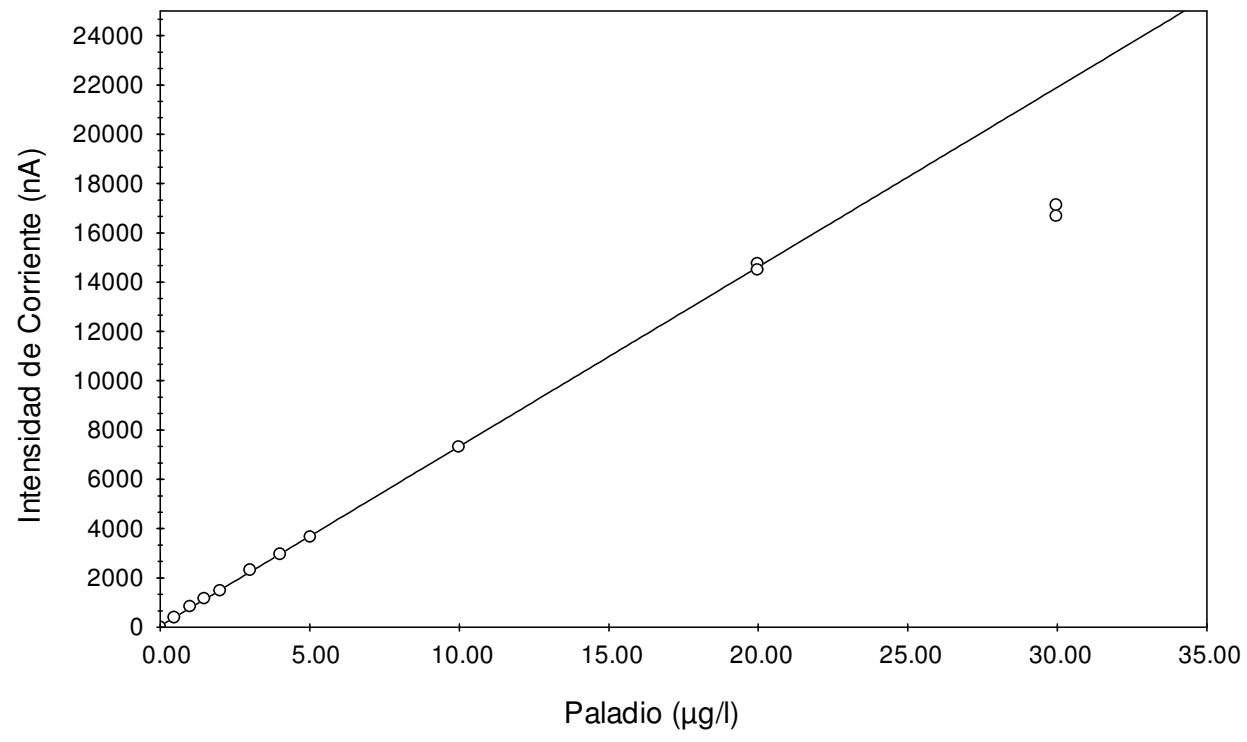

Figura 11. Linealidad de la determinación voltamperométrica de paladio.

\section{Estudio de linealidad}

Para el estudio de la linealidad del método, se colocaron en la celda voltamperométrica $10 \mathrm{~mL}$ de tampón, a los que se le agregó la dimetilglioxima. Sobre este electrolito soporte, se hicieron adiciones estándar de la disolución 
patrón, de forma que se provocara un aumento paulatino de la concentración. Los resultados se muestran en la Fig. 11.

De los resultados se puede concluir que la concentración máxima que se puede medir sin que se pierda la linealidad del método es $20 \mathrm{mg} / \mathrm{L}$ de paladio, cuando se utiliza un tiempo de preadsorción de 5 minutos.

\section{Estudio de interferencias}

Se investigó la influencia que presentan $\mathrm{Na}^{+}, \mathrm{K}^{+}, \mathrm{Mg}^{2+}, \mathrm{Ca}^{2+}, \mathrm{Zn}^{2+} \mathrm{y} \mathrm{Cu}^{2+}$, debido a que siempre se encuentran presentes en las muestras medioambientales. Los resultados indican que el método no se ve afectado por estos cationes incluso a concentraciones tan altas como $10 \mathrm{mg} / \mathrm{L}$. Tampoco $\mathrm{Al}^{3+}$, que forma parte de la mayoría de los convertidores catalíticos de los coches, es una interferencia en el análisis incluso a una concentración de $10 \mathrm{mg} / \mathrm{L}$. El cobalto tampoco interfiere con el análisis del paladio, aunque presenta una señal voltamperométrica a -0.74 $\mathrm{V}$, cuando la concentración de este catión es del orden de los $\mathrm{mg} / \mathrm{L}$. La señal aparece justo después de la correspondiente al dimetilglioximato de paladio y antes de la correspondiente a la dimetilglioxima libre. Una concentración de 10 $\mathrm{mg} / \mathrm{L}$ de cobalto no afecta los resultados obtenidos. En cuanto al níquel, presenta una señal a $-0.68 \mathrm{~V}$, muy próxima a la señal del paladio. De tal manera que una concentración de níquel de $1 \mathrm{mg} / \mathrm{L}$ no tiene efecto sobre la señal del paladio, aun cuando la señal está bien definida. Sin embargo, una concentración de níquel de $10 \mathrm{mg} / \mathrm{L}$ produce una señal muy alta que enmascara por completo la señal de paladio. Por lo tanto, no se puede determinar paladio cuando la concentración de níquel en la disolución supere los $10 \mathrm{mg} / \mathrm{L}$.

\section{Método operatorio recomendado}

Una vez establecidas las condiciones óptimas de operación, se propone el siguiente método operativo:

- Los electrolitos soportes y las muestras se preparan en la propia celda voltamperométrica en la que se va a proceder con el análisis, con la siguiente composición: 
$\checkmark 10 \mathrm{~mL}$ de tampón acetato $0.1 \mathrm{~mol} / \mathrm{L}$ y pH 3.6 ó, dependiendo del volumen de muestra a analizar, la cantidad necesaria del mismo para que el volumen final sea de $10 \mathrm{~mL}$;

$\checkmark$ dimetilglioxima para tener una concentración de $0.10 \mathrm{mmol} / \mathrm{L}$.

- Se procede al análisis utilizando las condiciones voltamperométricas que se resumen en la Tabla 4.

- La cuantificación se efectúa por el método de las adiciones estándar, haciendo por lo menos dos adiciones de $10 \mu \mathrm{L}$ de la disolución de paladio de $0.10 \mathrm{mg} / \mathrm{L}$ (incrementos de concentración de $0.1 \mu \mathrm{g} / \mathrm{L}$ ).

Tabla 4. Condiciones voltamperométricas óptimas para la determinación de paladio.

\begin{tabular}{|c|c|c|c|}
\hline Parámetro & Valor & Parámetro & Valor \\
\hline Gota & Grande $(\mathrm{L})$ & $\Delta \mathrm{E}$ & $-75 \mathrm{mV}$ \\
\hline $\mathrm{E}_{\text {preadsorción }}$ & $-0.30 \mathrm{~V}$ & Frecuencia & $50 \mathrm{~Hz}$ \\
\hline $\mathrm{t}_{\text {preadsorción }}$ & $0-600 \mathrm{~s}$ & Incremento & $10 \mathrm{mV}$ \\
\hline $\mathrm{t}_{\text {reposo }}$ & $5 \mathrm{~s}$ & Velocidad & $500 \mathrm{mV} / \mathrm{s}$ \\
\hline
\end{tabular}

\section{Reproducibilidad, límites de detección y de cuantificación}

Para la determinación de la reproducibilidad y de los límites de detección y de cuantificación, se prepararon $100 \mathrm{~mL}$ de tres disoluciones conteniendo cada una paladio:

- Disolución 1:

$0.155 \mu \mathrm{g} / \mathrm{L}$

- Disolución 2:

$0.369 \mu \mathrm{g} / \mathrm{L}$

- Disolución 3:

$$
2.07 \mu \mathrm{g} / \mathrm{L}
$$

Las tres disoluciones se analizaron individualmente por lo menos 7 veces cada una, utilizando las condiciones voltamperométricas establecidas. Los resultados de la reproducibilidad se muestran en la Fig. 12. 


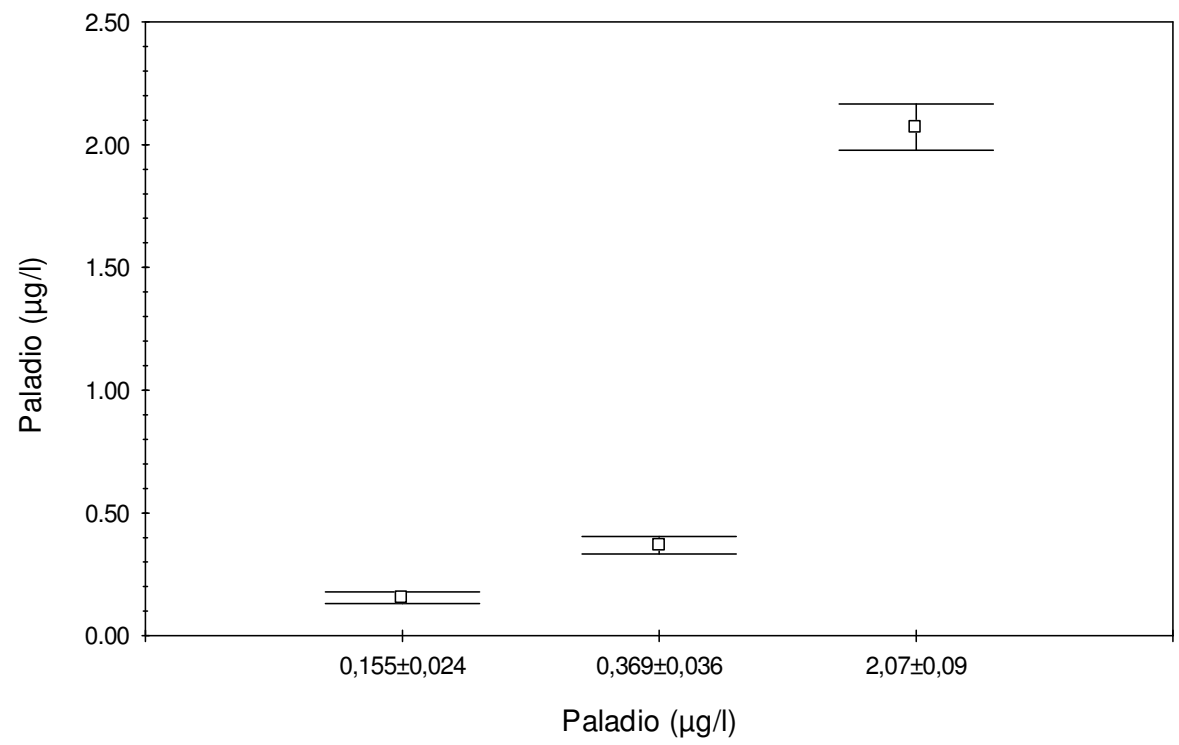

Figura 12. Reproducibilidad de la determinación de paladio a tres niveles de concentración.

Se puede ver como la desviación estándar absoluta de las determinaciones aumenta al aumentar la concentración de paladio en las disoluciones. Sin embargo, la desviación estándar relativa disminuye conforme aumenta la concentración de paladio y pasa de un $15.5 \%$ al nivel menos concentrado hasta un $4.3 \%$ a la mayor concentración. Es de notar, que estas desviaciones estándar son aceptables desde el punto de vista analítico, teniendo en cuenta la baja concentración de paladio que se está determinando.

En la Tabla 5 se muestran los límites de detección y de cuantificación del método. Estos se calcularon a partir de la recta de calibrado para paladio utilizando el método de las adiciones estándar y siguiendo el método de los límites de confianza $[12,13]$. Para ello, se interpoló en la recta de calibrado, el valor de intensidad de corriente en el intercepto con la ordenada presentado por la asíntota superior de los límites de confianza al 95\%, obteniéndose un valor que se considera como el límite de detección. A partir de éste y multiplicándolo por 10/3 se obtiene el límite de cuantificación. La Fig. 13 muestra una de las rectas de calibrado utilizadas en el cálculo de los límites. 
Tabla 5. Límites de detección y de cuantificación y sensibilidades de la determinación voltamperométrica de paladio.

\begin{tabular}{cc}
\hline & Paladio \\
\hline Límite de detección & $10 \mathrm{ng} / \mathrm{L}$ \\
Límite de cuantificación & $33 \mathrm{ng} / \mathrm{L}$ \\
Sensibilidad (nA.L/ng) & $(4.3 \pm 0.8) * 10^{2}$ \\
& $\mathrm{n}=7$ \\
\hline
\end{tabular}

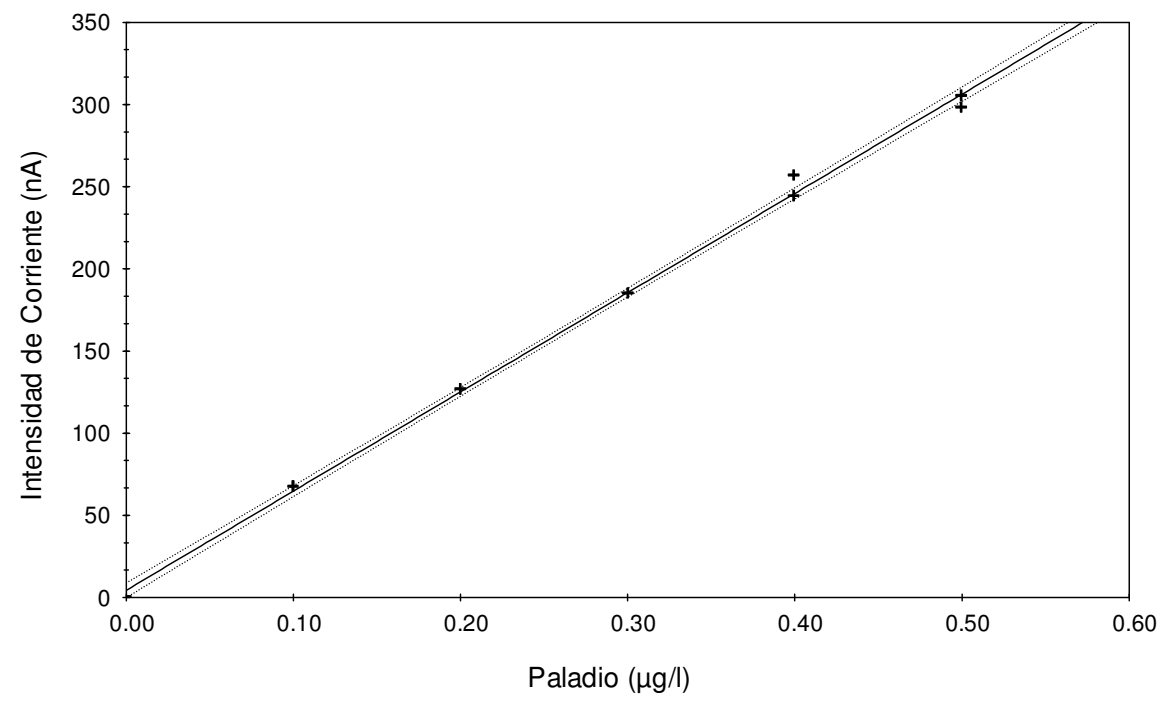

Figura 13. Establecimiento de los límites de detección y de cuantificación para la determinación de paladio.

\section{Características del método propuesto}

Como consecuencia del estudio realizado se puede concluir que:

- es un método de determinación de paladio aplicable a muestras con bajas concentraciones del metal;

- los riesgos de contaminación de la muestra son mínimos, debido a la facilidad de limpieza del material utilizado y a las bajas concentraciones de paladio ambientales existentes;

- se mejoran los límites de detección y de cuantificación para metodologías similares descritas en la literatura;

- no presenta interferencias significativas que puedan afectar los resultados, aparte del níquel que si interfiere; 
- presenta la desventaja de ser un método laborioso, lento y de difícil automatización.

\section{Agradecimientos}

Se agradece la colaboración de la Vicerrectoría de Investigación de la Universidad de Costa Rica, por el apoyo brindado a través del proyecto 804-A4093.

\section{Determinación de Ultratrazas de Paladio en Muestras Ambientales por Voltamperometría Adsortiva}

\section{Resumen}

El objetivo del presente trabajo es el estudio y optimización de una metodología voltamperométrica para determinar trazas y ultratrazas de paladio, con el fin de poder establecer los niveles ambientales de este elemento y verificar si se produce una contaminación a partir de los gases de escape de los vehículos equipados con convertidores catalíticos. La determinación voltamperométrica de paladio se fundamenta en la formación de un complejo del paladio con la dimetilglioxima, que se adsorbe activamente sobre la superficie de un electrodo de gota suspendida de mercurio, para luego ser reducido al efectuar el barrido de potencial con onda cuadrada. La cuantificación se efectúa por el método de las adiciones estándar. El estudio de interferencias demostró que $\mathrm{Na}^{+}, \mathrm{Mg}^{2+}, \mathrm{K}^{+}, \mathrm{Ca}^{2+}, \mathrm{Zn}^{2+}, \mathrm{Cu}^{2+}$ y $\mathrm{Al}^{3+}$ no son interferencias incluso al nivel de $10 \mathrm{mg} / \mathrm{L}$. Los límites de detección y de cuantificación de la metodología son $10 \mathrm{ng} / \mathrm{L}$ y $33 \mathrm{ng} / \mathrm{L}$ respectivamente, con una sensibilidad de $(4.3 \pm 0.8) * 10^{2} \mathrm{nA} \mathrm{L} / \mathrm{ng}$.

Palabras clave: paladio, ultratrazas, voltamperometría, determinación, ambientales.

\section{Referencias}

1. E. Helmers, K. Kümmerer, en: F. Zereini, F. Alt, (Eds), Anthropogenic Platinum-Group Element Emissions, Springer, Berlín, 2000.

2. E. Merian, (Ed.) Metals and Their Compounds in the Environment, VCH, Weinheim, 1991.

3. R. Barefoot, Trends Anal. Chem. 18 (1999) 702-707.

4. G. Philippeit, J. Angerer, J. Chromatography B 760 (2001) 237-245.

5. J. Messerschmidt, F. Alt, G. Tölg, J. Angerer, K. Schaller, Fresenius J. Anal. Chem. 343 (1992) 391-394. 
6. T. Gebel, en: F. Zereini, F. Alt, (Eds), Anthropogenic Platinum-Group Element Emissions, Springer, Berlín, 2000.

7. T. Kaiser, W. Schwarz, M. Frost, Einträge von Stoffen in Böden - eine Abschätzung des Gefährdungspotentials - Logos Verlag, Berlin, 1998.

8. T. Hees, B. Wenclawiak, S. Lustig, P. Schramel, M. Schwarzer, M. Schuster, D. Verstraete, R. Dams, E. Helmers, Environ. Sci. Pollut. Res. 5 (1998) 105-111.

9. D. Laschka, M. Nachtwey, en: F. Zereini, F. Alt, (Eds), Anthropogenic Platinum-Group Element Emissions, Springer, Berlín, 2000.

10. G. Raber, K. Kalcher, C. Neuhold, C. Talaber, G. Kölbl, Electroanalysis 7 (1995) 138-142.

11. M. Georgieva, B. Pihlar, Electroanalysis 12 (1996) 1155-1159.

12. J. Mandel, F. Linnig, Anal. Chem. 29 (1957) 743-749.

13. J.C. Miller, J.N. Miller, "Estadística para Química Analítica", 2a Ed., Addison-Wesley Iberoamericana: Delaware, 1993. 